\title{
The location of an active nucleus and a shadow of a tidal tail in the ULIRG Mrk 273
}

\author{
K. Iwasawa ${ }^{1}$, J. M. Mazzarella ${ }^{2}$, J. A. Surace ${ }^{3}$, D. B. Sanders ${ }^{4}$, L. Armus ${ }^{3}$, A. S. Evans ${ }^{5}$,

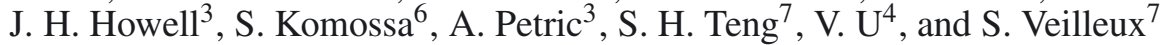

\author{
${ }^{1}$ ICREA and Institut de Ciències del Cosmos, Universitat de Barcelona (IEEC-UB), Martí i Franquès, 1, 08028 Barcelona, Spain \\ e-mail: kazushi.iwasawa@icc.ub.edu \\ 2 IPAC, California Institute of Technology, Pasadena, CA 91125, USA \\ 3 Spitzer Science Center, California Institute of Technology, Pasadena, CA 91125, USA \\ ${ }^{4}$ Institute for Astronomy, 2680 Woodlawn Drive, Honolulu, HI 96822-1839, USA \\ 5 Department of Astronomy, University of Virginia, 530 McCormick Road, Charlottesville, VA 22904 and NRAO, \\ 520 Edgemont Road, Charlottesville, VA 22903-2475, USA \\ ${ }^{6}$ Max Planck Institut für extraterrestrische Physik, Gießenbachstraße, 85748 Garching, Germany \\ 7 Department of Astronomy, University of Maryland, College Park, MD 20742, USA
}

Received 5 October 2010 / Accepted 17 January 2011

\section{ABSTRACT}

\begin{abstract}
Analysis of data from the Chandra X-ray Observatory for the double nucleus ULIRG Mrk 273 reveals an absorbed hard X-ray source coincident with the southwest nucleus, implying that this unresolved, near-infrared source is where an active nucleus resides, while the northern nuclear region contains a powerful starburst that dominates the far infrared luminosity. There is evidence of a slight image extension in the 6-7 keV band, where an Fe $\mathrm{K}$ line is present, towards the northern nucleus. A large-scale, diffuse emission nebula detected in soft X-rays contains a dark lane that spatially coincides with a high surface-brightness tidal tail extending $\sim 50$ arcsec $(40 \mathrm{kpc})$ to the south. The soft X-ray source is likely located behind the tidal tail, which absorbs X-ray photons along the line of sight. The estimated column density of cold gas in the tidal tail responsible for shadowing the soft X-rays is $N_{\mathrm{H}} \geq 6 \times 10^{21} \mathrm{~cm}^{-2}$, consistent with the tidal tail having an edge-on orientation.
\end{abstract}

Key words. galaxies: active - X-rays: galaxies - galaxies: individual: Mrk 273 - infrared: galaxies

\section{Introduction}

Mrk 273 (=UGC 8696, IRAS F13428+5608) is a nearby $(z=$ 0.0378 ) ultra luminous infrared galaxy (ULIRG) with a $8-$ $1000 \mu \mathrm{m}$ luminosity of $1.6 \times 10^{12} L_{\odot}$. The galaxy has a long tidal tail extending $\sim 40 \mathrm{kpc}$ to the south, indicating that the system is an ongoing merger. The nuclear region appears complex, and two nuclei with a projected separation of $\sim 1^{\prime \prime}$ have been revealed by infrared imaging (Majewski et al. 1993; Knapen et al. 1997; Scoville et al. 2000). In low-resolution radio images, three radio components are present (Condon et al. 1991), and the northern (N) and southwest (SW) components are identified with the two infrared nuclei, while the southeast (SE) component is likely a star cluster seen in the Hubble Space Telescope (HST hereafter) ACS and NICMOS images (Scoville et al. 2000). Mrk 273 N is a much stronger radio source with resolved structures in the high-resolution imaging (Knapen et al. 1997; Carilli \& Taylor 2000; Bondi et al. 2005). It is the site of a high concentration of molecular gas containing $1 \times 10^{9} M_{\odot}$ in a disk traced by $\mathrm{CO}(2-1)$ (Downes \& Solomon 1998). Combined with the radio continuum morphology, which indicates an abundance of supernovae and supernova remnants (Carilli \& Taylor 2000; Bondi et al. 2005), this region is likely to contain a powerful starburst. The 1-17 $\mu \mathrm{m}$ spectral energy distribution (SED) of the $\mathrm{N}$ and SW nuclei obtained by high-resolution imaging with the MIRLIN midinfrared camera on Keck (Soifer et al. 2000) suggests that strong
PAH features detected in the ISO SWS (Genzel et al. 1998) and Spitzer IRS (Armus et al. 2007) spectra originate in the $\mathrm{N} \mathrm{nu-}$ cleus, in agreement with a strong starburst.

The presence of an active nucleus (AGN) has been indicated by the optical spectrum (Khachikian \& Weedman 1974; Sanders et al. 1988), which is supported by the detection of [Nev] $\lambda 14.3 \mu \mathrm{m}$ and [OIv] $\lambda 25.9 \mu \mathrm{m}$ (Genzel et al. 1998; Satyapal et al. 2004; Armus et al. 2007) and an absorbed hard X-ray source with $N_{\mathrm{H}} \simeq 4 \times 10^{23} \mathrm{~cm}^{-2}$ (Iwasawa 1999; Risaliti et al. 2000; Balestra et al. 2005), which is variable (Teng et al. 2009). Scoville et al. (2000) suggest that the SW nucleus hosts the AGN because it is red and unresolved in the NICMOS $K$-band image. Although the SW component only has a very weak extended radio continuum, its 1-17 $\mu \mathrm{m}$ SED (Soifer et al. 2000) shows that the $\mathrm{SW}$ nucleus is comparable to or brighter at 3.4 and $10.3 \mu \mathrm{m}$ than the $\mathrm{N}$ nucleus, suggesting a hot/warm dust component. Integral field spectroscopy of the nuclear region by Colina et al. (1999) shows that Seyfert 2 type excitation with strong [OIII] $\lambda 5007$ is found at the SW nucleus, while the $\mathrm{N}$ nucleus shows characteristics of a LINER (in agreement with the classification of Veilleux et al. 1995). However, a point-like hard $\mathrm{X}$-ray source imaged by the Chandra X-ray Observatory (hereafter Chandra) has been identified with the $\mathrm{N}$ nucleus (Xia et al. 2002; González-Martín et al. 2006). In this paper, we re-examine this identification, as Chandra astrometry has been improved since the reprocessing of the data with the new alignment file 
in 2007. A combination of a re-analysis of the Chandra data and an accurate astrometry on the HST images enables us to locate the hard X-ray source more accurately.

On a larger scale, there is soft X-ray emission extending $\sim 30 \mathrm{kpc}$ to the south. In the same region, a long optical tidal tail with a similar length is seen. The optical surface brightness of this tidal tail is one of the highest observed in a large sample of interacting/merging galaxies (Mazzarella \& Boroson 1993), suggesting it has a rare edge-on orientation in the line of sight. There should be no direct physical connection between the optical and X-ray features however, because one is the remnant of a galaxy merger made of stars and the other is likely hot gas in a galactic-scale outflow ("superwind", e.g., Heckman et al. 1990).

Mrk 273 is in the C-GOALS sample for which general X-ray properties obtained from Chandra observations are presented in Iwasawa et al. (2011). Further detailed study of the Chandra data are found in Xia et al. (2002; see also Ptak et al. 2003; Grimes et al. 2005; Satyapal et al. 2004; González-Martín et al. 2006). In this article, we focus on 1) the hard X-ray imaging of the nuclear region; and 2) the large, southern soft X-ray nebula and its relation to the tidal tail.

The cosmology adopted here is $H_{0}=70 \mathrm{~km} \mathrm{~s}^{-1} \mathrm{Mpc}^{-1}$, $\Omega_{\Lambda}=0.72, \Omega_{\mathrm{M}}=0.28$, consistent with that adopted for other objects in the Great Observatories All Sky LIRG Survey (GOALS, Armus et al. 2009). Thus the luminosity distance of $D_{\mathrm{L}}=173 \mathrm{Mpc}$ and the angular scale of $0.77 \mathrm{kpc} \mathrm{arcsec}^{-1}$ are assumed.

\section{Observation and data reduction}

Mrk 273 was observed with Chandra on 2000 April 19 (Obs ID 809) using the focal plane detector ACIS-S operated in VFAINT imaging mode. The nucleus of the galaxy was placed at the off-axis angle of 1 arcmin on the detector. The useful exposure time is $44.2 \mathrm{ks}$. The data were reduced with the standard Chandra data analysis package CIAO 4.2 with the latest calibration files in CALDB 4.3.

We use the archival $H$ band image of Mrk 273 obtained from the HST-NICMOS with the NIC2 F160W filter (Scoville et al. 2000). The optical $B$ and $I$ band images were taken by the HSTACS with the $F 435 W$ and $F 814 W$ filters, respectively, as part of GOALS (Evans et al., in prep.).

The astrometry correction of the HST image was carried out with the Starlink software GAIA. The X-ray spectral analysis was performed using the HEASARC's FTOOLS and XSPEC version 12.

\section{Results}

\subsection{The position of the hard X-ray source}

The compact hard X-ray source is located where the soft X-ray emission is suppressed due to obscuration and thus the brightest soft X-ray region is displaced to the north by $\sim 1 \operatorname{arcsec}$ (see Iwasawa et al. 2011; Xia et al. 2002). We restrict the energy range of the image to $4-6 \mathrm{keV}$ band, where the image is close to point-like. Our aim here is to locate the hard X-ray source in the infrared image with updated astrometry. The accuracy in absolute astrometry of the standard processing of the Chandra data (after 2007 May) can be as good as 0.22 arcsec at the $68 \%$ limit ( 0.42 arcsec at the $90 \%$ limit) for bright sources observed near the aimpoint of the ACIS-S. However, the standard processing of a HST image may have a positional error up to 1 arcsec. Given the small projected separation $(\approx 1 \mathrm{arcsec})$ of the two nuclei, it is crucial to register the infrared and X-ray images as accurately as possible.

The HST-ACS I band image (Fig. 1a) is used as a reference for registering the near IR and the X-ray images as follows. The absolute astrometry of the ACS image has been corrected using field stars in the 2MASS catalog, which is only possible with the large field of view of the ACS and results in the astrometric accuracy to $\sim 0.2$ arcsec. The nuclear region of Mrk 273 in the $I$ band image is confused by extinction of the complex dust structures and the two nuclei are not clearly identified. For this reason, we use compact optical sources which are located outside the dusty nuclear region for aligning the NICMOS and Chandra images.

Three candidate star clusters near the nuclear regions, seen in both $I$ and $H$ bands, were used to align the NICMOS image (Fig. 1b) to the ACS image (Fig. 1a). These objects are labeled in Fig. 1a, and they may also be identified by their coordinates as GOALS J134442.17+555312.9 (CSC1), J134442.11+555314.5 (CSC2), and J134442.15+555312.5 (CSC3). Since two of the star clusters are faint, the relative accuracy of the alignment is estimated to be about their beam size $\sim 0.15 \operatorname{arcsec}^{1}$.

The Chandra image was aligned using the bright X-ray source Mrk 273X (see Fig. 1e), a Seyfert 2 galaxy at $z=0.458$ (Xia et al. 1998), which is listed in the Chandra Multiwavelength Project catalog as CXOMP J134447.4+555411 (Kim et al. 2007) and its optical counterpart is marked in Fig. 1d. The X-ray source is point-like and has $\approx 1000$ counts in the $0.4-5 \mathrm{keV}$ band, with which the X-ray centroid is well determined by the detection algorithm celldetect. The X-ray position was displaced by $0.1 \mathrm{arcsec}$ to the east from the optical nucleus of the galaxy, and a correction has been applied to the X-ray astrometry. The centroid position of the $4-6 \mathrm{keV}$ source is then found to be (RA, Dec $)_{\mathrm{J} 2000}=\left(13^{\mathrm{h}} 44^{\mathrm{m}} 42.06^{\mathrm{s}},+55^{\mathrm{d}} 53^{\mathrm{m}} 12.77^{\mathrm{s}}\right)$.

After these registrations, the accuracy of the relative alignment between the NICMOS and Chandra images is expected to be $<0.2$ arcsec. Kim et al. (2007) derived empirical formulae for positional uncertainty for a Chandra source as a function of source brightness and off-axis angle of the source location, using image simulations. As this uncertainty is derived from displacements between the input and simulated image positions, it can be considered as a statistical error. The hard X-ray source has $362 \pm 9$ counts in the $4-6 \mathrm{keV}$ band. The uncertainty estimated from the relevant formula is $0.25 \operatorname{arcsec}(1 \sigma)$. We thus adopt 0.35 arcsec as the absolute astrometric error. The position of the hard X-ray source and the error circle are indicated in the NICMOS image (Fig. 1b), which shows that the hard X-ray source is likely associated with the red, near-infrared SW nucleus (Fig. 1c).

\subsection{The SE radio component}

Figure 1c shows an overlay of the VLA $3.6 \mathrm{~cm}$ image contours (Condon et al. 1991) on the composite ACS/NICMOS image. No astrometry correction has been applied to the radio image. There is a slight offset of the radio image by $\sim 0.15$ arcsec to the south, which appears to be a remaining alignment error, as the SW radio component has a similar shift relative to the near-infrared nucleus. As shown in Fig. 1c, the SE radio component is likely associated with a star cluster seen in the ACS and NICMOS images, as first pointed out by Scoville et al. (2000). High resolution radio imaging shows that this source is extended and has a

\footnotetext{
1 In the astrometry corrected NICMOS image, the 2MASS source is located between the two near IR nuclei, which is likely an artifact of the low resolution of the 2 MASS survey.
} 


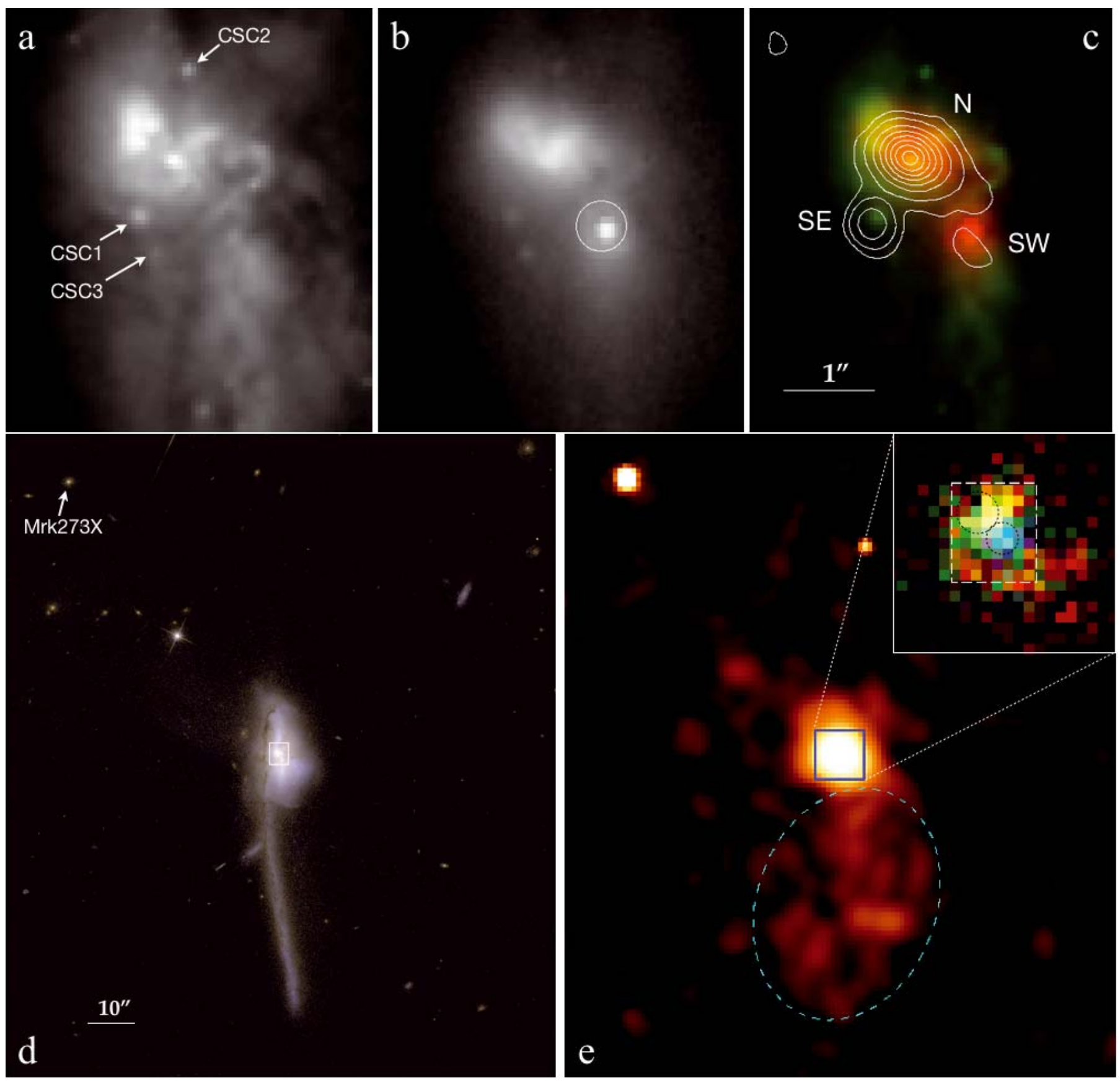

Fig. 1. a) The HST-ACS $I$ band $(F 814 W)$ image of the nuclear region of Mrk 273. Candidate star clusters used to register the NICMOS image with the ACS image are labeled in order of their luminosity. The orientation of this and all the other images is north up, east to the left. b) The HST-NICMOS $H$-band $(F 160 \mathrm{~W})$ image of the same region as in a). The point-like SW nucleus, which is not evident in the optical band, is clearly visible. The centroid of the hard X-ray (4-6 keV) source is indicated by the error circle of 0.35 arcsec radius. c) The composite of the $I$-band (green) and $H$-band (red) images of the same region as in $\mathbf{a}$ ) and b), overlaid by the VLA $8.4 \mathrm{GHz}$ image (Condon et al. 1991) in contours. No astrometric correction has been applied to the VLA image. The three radio components, N, SE and SW are labeled. The lowest contour represents $8 \%$ level of the peak brightness at the $\mathrm{N}$ component and other contours increases by factors of 3.3. The scale bar indicates 1 arcsec $(=0.77 \mathrm{kpc})$. d) The HST-ACS $B(F 435 W)+I(F 814 W)$ composite image of a large field of view $\left(2.0^{\prime} \times 2.3^{\prime}\right)$ around Mrk 273 . The area of the images for the nuclear region in a), b) and $\mathbf{c})$ is indicated by a white rectangle. The bright, point-like X-ray source Mrk $273 \mathrm{X}$, a distant $(z=0.46)$ Seyfert 2 galaxy, seen in the X-ray image (Fig. 1e) and used to register the Chandra image with the ACS image is labeled. The scale bar indicates 10 arcsec $(=7.7 \mathrm{kpc})$. e) The Chandra ACIS-S 0.4-7 keV image of the same area of the sky as in d). Details of the bright, central part are shown in the inset: three colour composite of unsmoothed images of the $0.4-1.1 \mathrm{keV}$ (red), 1.1-3 keV (green) and 3-7 keV (blue) bands for the 10" $\times 10^{\prime \prime}$ region, as marked in the main figure. The two black, dashed circles indicate the regions where the spectral data (N and SW) shown in Fig. 3 were taken. The SW region is centred on the hard X-ray source, where the X-ray colour is the bluest. The white dashed rectangle indicates the region for a), b) and c). The ellipse in the main figure indicates the region where the spectral data for the southern X-ray nebula (Fig. 5) were collected. 

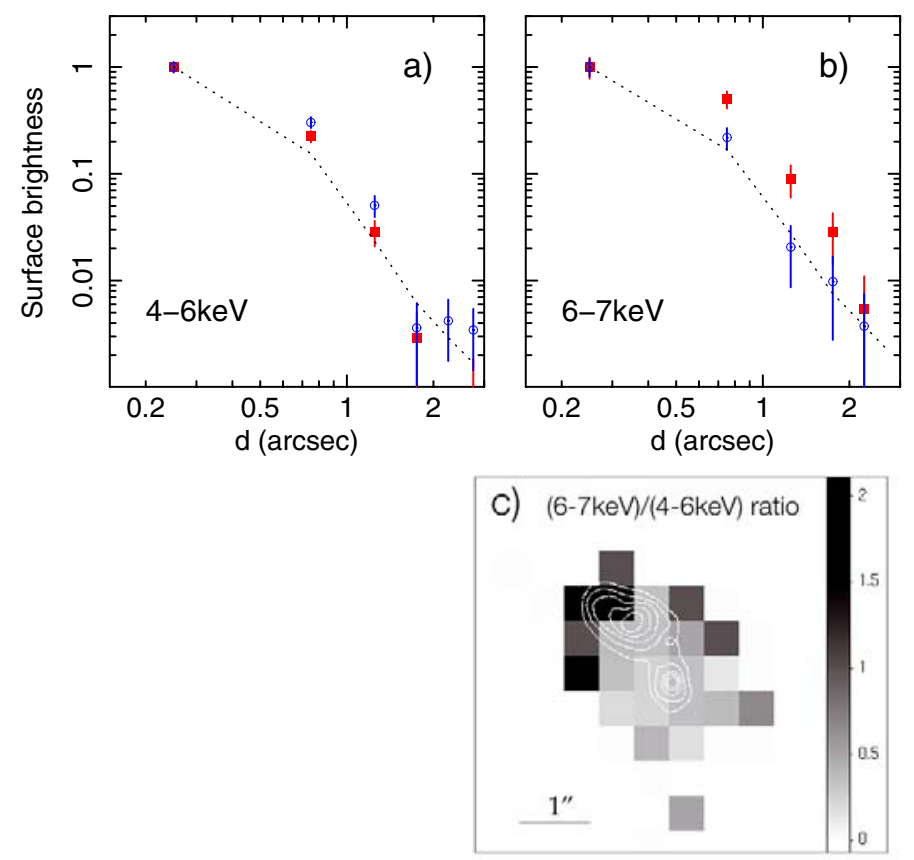

Fig. 2. a) The radial surface brightness profiles of the NE (squares) and SW (circles) halves in the 4-6 keV band, with the simulated PSF at $4.51 \mathrm{keV}$ (dotted line); b) same as a) but in the 6-7 keV band, with the similated PSF at $6.4 \mathrm{keV}$ (dotted line); c) The (6-7 keV)/(4-6 keV) ratio image of the nuclear region of Mrk 273, overlaid by the NICMOS $\mathrm{H}$-band image contours showing the $\mathrm{N}$ and $\mathrm{SW}$ nuclei. The contours are drawn at five logarithmic intervals with the lowest level at $20 \%$ intensity of the peak brightness at the centre of the SW nucleus. The orientation of the image is north up, east to the left. The scale bar indicates 1 arcsec. This map shows a trend of a 6-7 keV band enhancement towards NE.

steep spectrum (Carilli \& Taylor 2000; Bondi et al. 2005). These radio observations suggest a starburst as its origin (Bondi et al. 2005) but they pointed out that the lack of a near-IR counterpart was a problem. An association of the radio source with the star cluster seen in optical and near infrared would favour a starburst interpretation (further discussed in Sect. 4.1).

\subsection{Possible extension of 6-7 keV emission}

The 4-6 keV emission, used in the imaging analysis above, is dominated by an absorbed continuum of the active nucleus with negligible contribution from extended emission seen at lower energies. Figure 2 a shows the radial profiles of the $4-6 \mathrm{keV}$ emission in the northeast $\left(\mathrm{PA}=45^{\circ}\right)$ and southwest $\left(\mathrm{PA}=225^{\circ}\right)$ halves, indicating the $4-6 \mathrm{keV}$ source is approximately symmetric in the northeast-southwest direction and close to the point spread function (PSF). However, in the 6-7 keV band (Fig. 2b), the northeast half shows a $\sim 3 \sigma$ excess over the southwest half, which is consistent with the PSF. This excess carries about $10 \%$ of the total 6-7 keV emission (121 counts) and is found around the $\mathrm{N}$ nucleus, as shown in the $(6-7 \mathrm{keV}) /(4-6 \mathrm{keV})$ ratio image (Fig. 2c).

The 6-7 keV band contains the $6.4 \mathrm{keV}$ Fe $\mathrm{K}$ line (at $6.17 \mathrm{keV}$ in the observed frame). The spectrum taken from a 1 " diameter aperture of the 6-7 keV excess region, as indicated in Fig. 1e (inset), indeed shows a strong $6.4 \mathrm{keV} \mathrm{Fe} \mathrm{K}$ line with equivalent width, $E W=1.0 \pm 0.5 \mathrm{keV}$ (labeled as "N" in Fig. 3). For a comparison, another spectrum was taken from a region centred on the SW nucleus with a $1.5^{\prime \prime}$ diameter aperture (as indicated in Fig. 1e inset), which does not overlap the

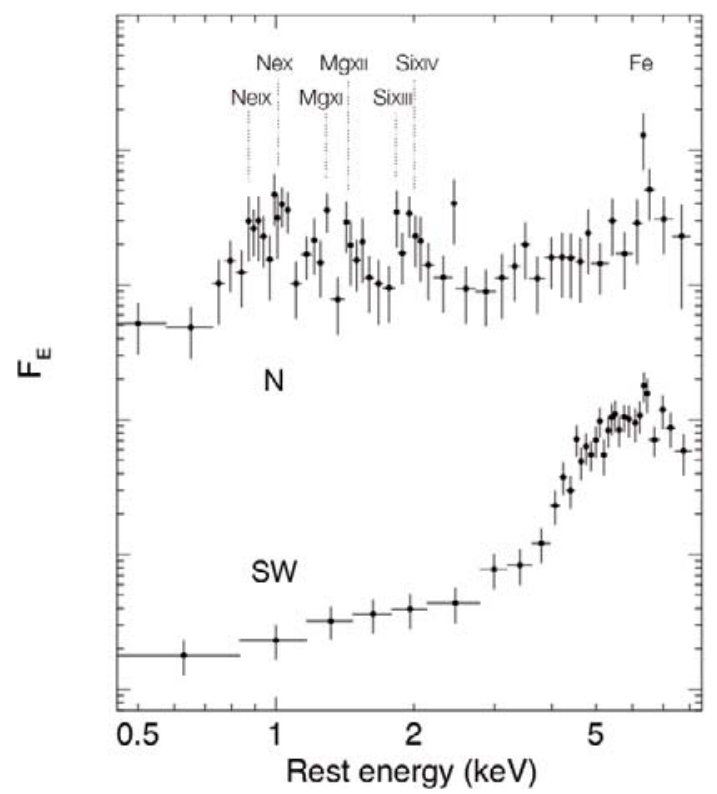

Fig. 3. The Chandra ACIS-S spectra of the 6-7 keV excess region (Fig. 2c) at the $\mathrm{N}$ nucleus and of the SW nucleus. The spectral apertures are 1 arcsec and 0.75 arcsec in radius, respectively, and are shown in Fig. 1e (inset). The spectra are presented in flux density unit and arbitrarily shifted for visual clarity, and the energy scale has been corrected for the galaxy redshift. The $\mathrm{N}$ spectrum shows a possibly larger EW of $\mathrm{Fe} \mathrm{K}$ at $6.4 \mathrm{keV}$ than the SW spectrum and the soft X-ray lines indicating high ionization (e.g., $\mathrm{H}$-like $\mathrm{Mg}$ and $\mathrm{Si}$ ), as marked.

above 6-7 keV excess region. This spectrum (labeled as " $\mathrm{SW}$ " in Fig. 3) is essentially of the absorbed active nucleus and the $6.4 \mathrm{keV}$ Fe $\mathrm{K}$ line has $E W=0.23_{-0.07}^{+0.09} \mathrm{keV}$, which agrees with that measured for the total emission of the objects (Ptak et al. 2003; Xia et al. 2002; Iwasawa et al. 2011). Thus the Fe K line in the $\mathrm{N}$ spectrum is much stronger (with respect to the contiuum) than in the SW spectrum.

This is consistent that the $6-7 \mathrm{keV}$ excess is mainly due to an enhancement of the Fe K line, suggesting possible presence of an extra source of $\mathrm{Fe} \mathrm{K}$, albeit the evidence is marginal given the limited statistics. If it is real, a reflection-dominated spectrum, as seen in a heavily obscured AGN, is a good candidate for this extra source, since it contributes primarily to the Fe $\mathrm{K}$ line but significantly less to the continuum. The $3-8 \mathrm{keV}$ continuum of the $\mathrm{N}$ spectrum is flat $\left(\Gamma=0.0_{-0.6}^{+0.4}\right)$ and shows no obvious sign of an absorption cut-off, as seen in the SW spectrum. This is also consistent with a reflection spectrum but the absorption cut-off in the lower energies may be masked by strong soft X-ray emission. The reality of the image extension and the Fe line enhancement there need to be investigated further with higher quality (both in spatial resolution and counting statistics) data. The Fe K excess region spatially coincides with the $\mathrm{N}$ nucleus, which could lead to an interesting possibility, as discusse below (Sect. 4.1).

This region is also brightest in soft X-rays, particularly in the 1-3 keV band (Fig. 1e inset). The soft X-ray spectrum shows emission-lines of $\mathrm{H}$-like $\mathrm{Ne}, \mathrm{Mg}$ and $\mathrm{Si}$ that are comparable or even stronger than their He-like counterparts (as marked in Fig. 3), indicating high-ionization. If these lines are of thermal origin, the temperature should be $k T \simeq 1.5 \pm 0.3 \mathrm{keV}$, as inferred by a thermal spectrum fit to the energy range of the $\mathrm{Mg}$ and $\mathrm{Si}$ lines. This temperature is much higher than the mean temperature of the bright central part, e.g., inner $10^{\prime \prime}$ area $(k T \sim 0.8 \mathrm{keV}$, $\mathrm{Xia}$ et al. 2002; Grimes et al. 2005). Note that $\mathrm{Ne}$ IX $(0.9 \mathrm{keV})$ is 


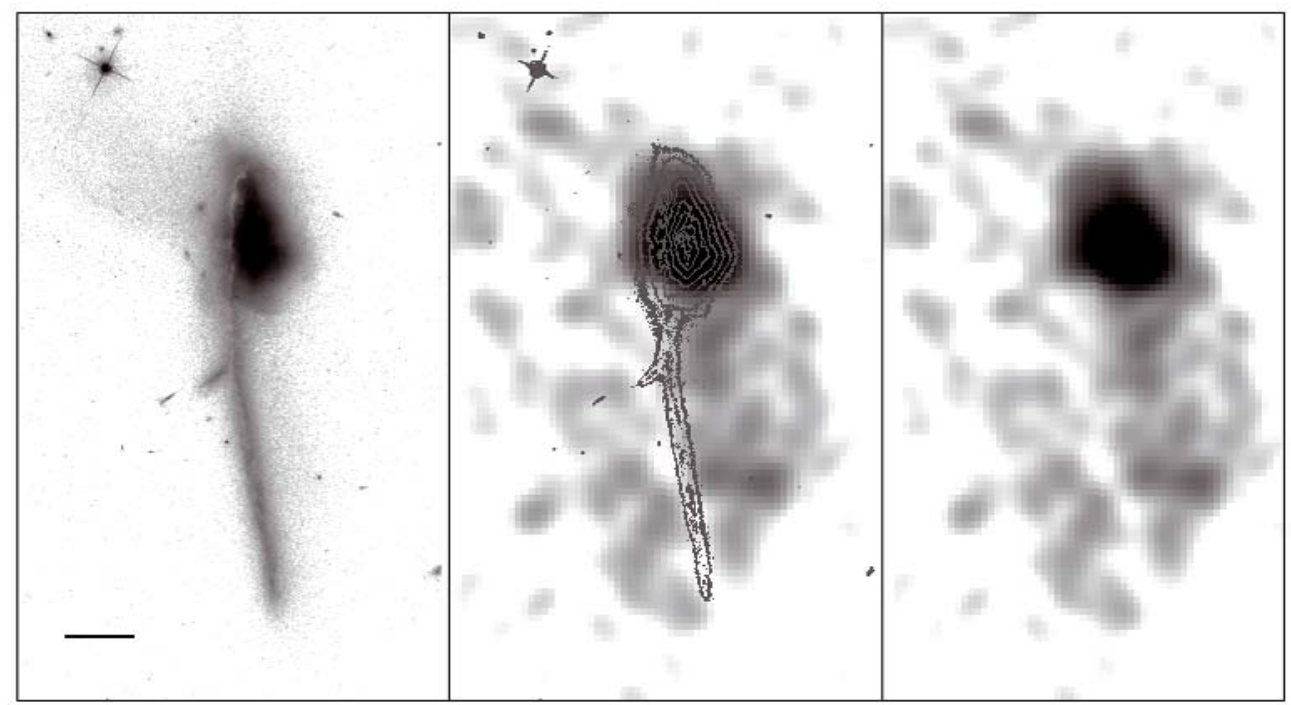

Fig. 4. Left: the HST-ACS I-band $(F 814 W)$ image of Mrk 273. Middle: the 0.4-1.1 keV Chandra image of Mrk273, overlaid by the contours obtained from the optical I-band image; Right: the same 0.4-1.1 keV Chandra image as in the middle panel. The X-ray image of 1 arcsec resolution has been smoothed using a circular Gaussian kernel with the dispersion of 1.8 arcsec. The contours for the HST optical image are drawn at 10 logarithmic intervals. In the right panel, a dark lane runs through the soft X-ray nebula in the N-S direction, which coincides with the area where the optical tidal tail is preent. The orientation of the images is north up, east to the left. The scale bar corresponds to 10 arcsec.

not expected at $k T \sim 1.5 \mathrm{keV}$, and its detection perhaps suggests the presence of multi-temperature gas in this region.

\subsection{The extended soft $X$-ray nebula}

\subsubsection{A shadow of the tidal tail}

A large soft X-ray nebula extending to the south is clearly visible in the Chandra image (Fig. 1e), as noted by several authors (Xia et al. 2002; González-Martín et al. 2006; Ptak et al. 2003; Grimes et al. 2005). Comparison of the soft X-ray and optical images shows that the long, optical tidal tail extending to the south is remarkably well aligned with a dark gap running through the soft $\mathrm{X}$-ray nebula in the N-S direction (Fig. 4). The position angle (PA) of the X-ray dark lane is $\sim 10^{\circ}$, which increases to $\sim 15^{\circ}$ at the southern end while the optical tidal tail has PA $\sim 10^{\circ}$. This can be understood if the tidal tail is located in front of the soft $\mathrm{X}$-ray nebula in our line of sight such that soft X-ray emission is absorbed by the cold gas in the tidal tail.

The ACIS-S spectrum of the southern X-ray nebula (Fig. 5), taken from the region indicated in Fig. 1e, shows that the bulk of the light is emitted below $1.1 \mathrm{keV}$. The total source counts in the $0.4-1.1 \mathrm{keV}$ band are $\sim 300$ counts. To make emission of such a spectrum totally suppressed at energies below $1 \mathrm{keV}$, an absorbing column density of $N_{\mathrm{H}} \approx 1 \times 10^{22} \mathrm{~cm}^{-2}$ or larger is required. However, as the X-ray source is diffuse, the limiting column density would be slightly relaxed. The projected area of the tidal tail's shadow is measured to be $\approx 2.5 \times 38 \operatorname{arcsec}^{2}$. Under a crude assumption of a uniform brightness distribution over the nebula, we adopt detection of $2 \sigma$ counts in more than half the area is the limiting condition. Assuming the spectral model for the nebula (Sect. 3.4.2), expected counts from the shadowed area are simulated for incremental $N_{\mathrm{H}}$ values of absorbing matter at the galaxy redshit. As $N_{\mathrm{H}}$ is increased by an interval of $\Delta N_{\mathrm{H}}=1 \times 10^{21} \mathrm{~cm}^{2}$, expected counts go below the detection limit, when $N_{\mathrm{H}}$ exceeds $6 \times 10^{21} \mathrm{~cm}^{2}$. We consider this $N_{\mathrm{H}}$ as the lower limit of the column density of cold gas in the tidal tail, which makes the shadow on the soft X-ray nebula. This column density is similar to that of an edge-on galaxy disk. Similar

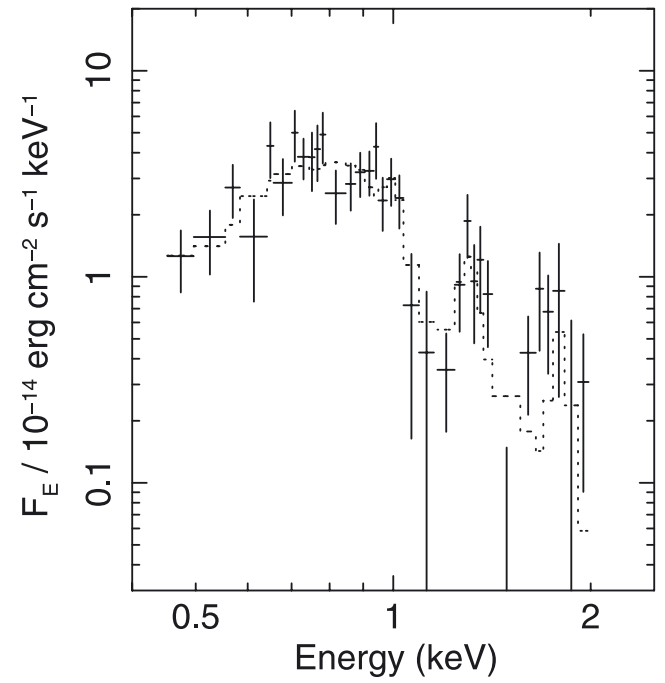

Fig. 5. The ACIS-S spectrum of the southern X-ray nebula of Mrk 273. The dotted-line histogram shows the best-fit thermal emission spectrum (see text for details). Note strong emission-lines from $\mathrm{Mg}$ XI and Si XIII in the $1-2 \mathrm{keV}$ range.

measurements of disk shadowing have been made against the extragalactic X-ray background (e.g., Barber et al. 1996).

\subsubsection{Spectral properties}

While the spectrum of the nebula (Fig. 5) is dominated by the soft X-ray emission below $1 \mathrm{keV}$, there is weak but significant emission up to $2 \mathrm{keV}$ containing $\sim 50 \pm 10$ counts, mainly due to line emission of $\mathrm{Mg}$ XI at $1.34 \mathrm{keV}$ and Si XIII at $1.85 \mathrm{keV}$. There is also an excess on the lower energy side of Si xIII. These strong lines suggest an enhancement of metallicity in $\alpha$ elements. Fitting the spectrum with a thermal emission model computed by MEKAL (Kaastra 1992), in which the abundances of elements relavant to the $0.4-2 \mathrm{keV}$ band are left variable, gives a metallicity of $Z(\mathrm{Ne}, \mathrm{Mg}, \mathrm{Si})=(3 \pm 1) Z_{\odot}, Z(\mathrm{Fe})=(0.4 \pm 0.3) Z_{\odot}$, 
and $Z(O)=(0.7 \pm 1) Z_{\odot}$ with a temperature of $k T=0.47_{-0.05}^{+0.08} \mathrm{keV}$ and the Galactic absorption $N_{\mathrm{H}}=9 \times 10^{19} \mathrm{~cm}^{-2}$ (Kalberla et al. 2005). The relatively small $\mathrm{Fe}$ and $\mathrm{O}$ abundances reflect weakness of the $\mathrm{Fe} \mathrm{L}$ emission bump around $1 \mathrm{keV}$ and $\mathrm{O}$ VIII at $0.65 \mathrm{keV}$. This result agrees with Grimes et al. (2005), but differs from the low metallicity obtained by Xia et al. (2002) who used data only below $1 \mathrm{keV}$, where the $\mathrm{Mg}$ and $\mathrm{Si}$ lines are not present, and with the solar abundance pattern.

Enhancement of metallicity in $\alpha$ elements is expected in the interstellar medium enriched by ejecta of core-collapse supernovae, which a starburst would mainly produce. The nebular spectrum is similar to that found in the extended nebula of another ULIRG Mrk 231 in terms of temperature and the strong $\mathrm{Mg}$ and Si lines (Iwasawa et al. 2011). The apparently lower metallicity of $\mathrm{O}$ than the other $\alpha$-elements is not compatible with the classical theoretical prediction (e.g., Nomoto et al. 1997) but similar to that found in well studied starbursts in NGC 6240 (Netzer et al. 2005) and M 82 (Ranalli et al. 2008).

\section{Discussion}

\subsection{Nature of the double nucleus}

With the registration with the HST image, the SW nucleus appears to be the location of the hard X-ray source in Mrk 273, which is absorbed by cold gas of $N_{\mathrm{H}} \sim 4 \times 10^{23} \mathrm{~cm}^{-2}$. This agrees with the suggestion by Scoville et al. (2000) that the SW nucleus, a red, unresolved NICMOS source, is the location of an active nucleus and other properties described in Sect. 1. Besides the Seyfert 2 spectrum at the SW nucleus, Colina et al. (1999) also pointed out that the kinematically quiescent [OIII] component, located $\sim 2^{\prime \prime}$ further SW of the SW nucleus, is similar to the ionization-cones around Seyfert 2 galaxies. The midIR AGN indicator [ $\mathrm{Ne}$ v] may therefore originate from this region. The mid-infrared to hard X-ray ratio, $\log L_{12 \mu \mathrm{m}} / L_{6 \mathrm{keV}} \simeq$ 1.2 , of the SW nucleus is comparable to those normally observed in AGN (e.g., Elvis et al. 1994; Lusso et al. 2010). While the radio emission is weak, the ratio of flux densities $f_{v}(12.5 \mu \mathrm{m}) / f_{v}(8.4 \mathrm{GHz})=290$, as derived by Soifer et al. (2000), is not unusual for a radio-quiet AGN.

The far-infrared portion of the SEDs of the two nuclei are not known. The radio band is dominated by the $\mathrm{N}$ component, with $\log P_{1.4 \mathrm{GHz}} \simeq 27.7 \mathrm{~W} \mathrm{~Hz}^{-1}$. If the far infrared emission, with $\log P_{70 \mu \mathrm{m}} \simeq 29.9 \mathrm{~W} \mathrm{~Hz}^{-1}$ (estimated by an interpolation of the IRAS $60 \mu \mathrm{m}$ and $100 \mu \mathrm{m}$ fluxes of Sanders et al. 2003), is also dominated by the $\mathrm{N}$ component, then the $1.4 \mathrm{GHz}$ and $70 \mu \mathrm{m}$ luminosities are consistent with the well-known farinfrared/radio correlation (e.g., Appleton et al. 2004). This suggests that the $\mathrm{N}$ nucleus is likely powered by a starburst and the major source of the far-infrared emisison where the bolometric power peaks $(\sim 60 \mu \mathrm{m})$. A similar conclusion can be reached by an argument based on the AGN luminosity of the SW nucleus. The absorption-corrected 2-10 keV luminosity of the hard X-ray source is $0.9 \times 10^{43} \mathrm{erg} \mathrm{s}^{-1}$. Assuming the bolometric correction of Marconi et al. (2004), the bolometric luminosity of the AGN in the SW nucleus is estimated to be $2 \times 10^{44} \mathrm{erg} \mathrm{s}^{-1}$ or $\log L_{\mathrm{AGN}}^{\mathrm{bol}} / L_{\odot} \sim 10.7$, which is a factor of $\sim 30$ smaller than the total $L_{\mathrm{IR}}$. Relative to this X-ray estimate, the mid-IR diagnostics with [Ne v] $\lambda 14.3 \mu \mathrm{m}$, [O IV] $\lambda 25.9 \mu \mathrm{m}$ and PAH emission at $6.2 \mu \mathrm{m}$ seem to suggest a larger (but not a dominant) contribution from AGN (Armus et al. 2007; Petric et al. 2010).

Downes \& Solomon (1998) suggested that the N nucleus is an extreme compact starburst with a high luminosity-density, similar to the western nucleus of Arp 220. High-resolution radio continuum imaging supports this hypothesis (Carilli \& Taylor 2000; Bondi et al. 2005). The LINER-type excitation and the adjascent, kinematically turbulent [OIII] component (Colina et al. 1999) can be explained by a strong outflow from the starburst. As discussed above, the total far-infrared output of Mrk 273 is likely dominated by a starburst occuring in this region. As shown in Fig. 1e, bright soft X-ray emission is observed here. The soft $\mathrm{X}$-ray spectrum shows thermal characteristics and high metallicity (Xia et al. 2002; Ptak et al. 2003; Grimes et al. 2005).

At energies above $4 \mathrm{keV}$, any contribution of the $\mathrm{N}$ nucleus appears to be negligible. A possible exception is in the 6-7 $\mathrm{keV}$ range. If the image extension in this energy range is real and due to enhanced $\mathrm{Fe} \mathrm{K}$ emission, as discussed in Sect. 3.3, this presents a possibility that a heavily absorbed (possibly Compton-thick) AGN may exist in the $\mathrm{N}$ nucleus as well. Assuming a reflection-dominated spectrum with a $6.4 \mathrm{keV} \mathrm{Fe} \mathrm{K}$ line with $E W=1 \mathrm{keV}$, an expected $4-7 \mathrm{keV}$ flux of this (hypothetical) source would be $\sim 2 \times 10^{-14} \mathrm{erg} \mathrm{s}^{-1} \mathrm{~cm}^{-2}$, which is only $5 \%$ of the total observed flux from the whole system in the same band. Even if an intrinsic source flux is 2 orders of magnitude larger, its 2-10 keV luminosity would be about $10^{43} \mathrm{erg} \mathrm{s}^{-1}$, similar to that of the SW and, in any case, have a minor contribution to the bolometric luminosity. It should be noted that the $6-7 \mathrm{keV}$ excess could also be due to extended Fe K emission induced by AGN in the SW nucleus, as seen in NGC 4388 (Iwasawa et al. 2003), rather than originating from the $\mathrm{N}$ nucleus. At the current data quality, evidence for an AGN in the $\mathrm{N}$ nucleus is thus weak. However, this raises an exciting possibility that Mrk 273 is another dual AGN system similar to the cases of NGC 6240 (Komossa et al. 2003) and Mrk 266 (NGC 5256, Mazzarella et al. 2011).

\subsection{Cold gas in the tidal tail shadowing soft $X$-ray nebula}

A rough estimate of the mass of cold gas in the tidal tail responsible for shadowing the soft X-rays can be obtained from the minimum column density $\left(N_{\mathrm{H}}^{\mathrm{lim}}=6 \times 10^{21} \mathrm{~cm}^{-2}\right)$ which is required to absorb away the soft $\mathrm{X}$-ray photons from the nebula (Sect. 3.4.1). If cold gas is distributed uniformly in a cylinder with a diameter of $2.5 \operatorname{arcsec}(d \sim 2 \mathrm{kpc})$ and a length of 40 arc$\sec (l \sim 30 \mathrm{kpc})$ lying on the lane of the sky, the mean density would be $n=N_{\mathrm{H}}^{\mathrm{lim}} / d \sim 1 \mathrm{~cm}^{-3}$. Thus the gas mass is estimated to be $\geq 2 \times 10^{9} M_{\odot}$. However, a more likely geometry of this tidal tail is an edge-on disk, as argued in Sects. 1 and 3.4. In this case, the average density would be lower.

Similarity in morphology between the soft X-ray and $\mathrm{H} \alpha$ emission has been noted by Xia et al. (2002) and Grimes et al. (2005). This correspondence is often found in objects with galactic-scale outflows, such as Arp 220 and NGC 6240 (e.g., Veilleux et al. 2005; Armus et al. 1990). The enhancement of $\alpha$-elements in the soft X-ray emitting gas (Sect. 3.3.2) supports prior interpretation of the diffuse nebula as a starburst-driven superwind. Although the outflow velocity at inner radii is as large as $2400 \mathrm{~km} \mathrm{~s}^{-1}$ (Colina et al. 1999), it will slow down quickly until escaping from the galaxy's potential. To reach $30 \mathrm{kpc}$, which is roughly the projected length of the southern extension of the nebula, the required time is $t \sim 10^{8}\left(v_{300}\right)^{-1} \mathrm{yr}$, where $v_{300}$ is the average outflow velocity in unit of $300 \mathrm{~km} \mathrm{~s}^{-1}$. This can be a crude measure of the starburst age. The linearity, thinness and high surface-brightness suggests that a tidal tail (remnants of a galaxy disk) is being viewed nearly edge-on, trailing a northward orbital encounter. Assuming the component of the relative orbital velocity in the plane of the sky is $\sim 300 \mathrm{~km} \mathrm{~s}^{-1}$, material 
in the tidal tail $\sim 30 \mathrm{kpc}$ south of the nuclei would have been drawn out from the parent galaxy disk $\sim 10^{8} \mathrm{yr}$ ago. If the starburst was episodic or roughly continuos over the duration of the ongoing merger, as suggested by simulations, the alignment of the diffuse X-ray emitting nebula and the stellar tidal tail is not a coincidence, but rather an artifact of the main power source of the starburst-driven wind moving northward during the creation of the trailing tidal tail.

\section{Summary}

With the new registration of the Chandra X-ray image with the optical and near-IR images from HST, we identified the absorbed, hard X-ray source of the Seyfert 2 nucleus in Mrk 273 with the SW nucleus, which revises the previous identification to the $\mathrm{N}$ nucleus. The hard $\mathrm{X}$-ray source is point-like except in the 6-7 keV band, where the image shows a slight extension to the northeast. This excess appears to be due to enhanced Fe $\mathrm{K}$ line emission at $6.4 \mathrm{keV}$ around the $\mathrm{N}$ nucleus. A possible implication is that a heavily obscured active nucleus might also be present in the $\mathrm{N}$ nucleus, while a starburst remains to be a dominant source of the far-IR luminosity.

A dark lane in the soft X-ray nebula extending to the south is found to align well with the optical tidal tail. This probably means the tidal tail is located in front of the soft X-ray nebula and absorbs the X-ray photons along our line of sight. The column density of cold gas in the tidal tail is estimated to be $N_{\mathrm{H}} \geq 6 \times$ $10^{21} \mathrm{~cm}^{-2}$.

Acknowledgements. This research made use of archival data maintained at the Chandra X-ray Center (CXC) and Space Telescope Science Institute (STScI), data products from the Two Micron All Sky Survey (2MASS), and the NASA/IPAC Extragalactic Database (NED).

\section{References}

Appleton, P. N., Fadda, D. T., Marleau, F. R., et al. 2004, ApJS, 154, 147 Armus, L., Heckman, T. M., \& Miley, G. K. 1990, ApJ, 364, 471

Armus, L., Charmandaris, V., Bernard-Salas, J., et al. 2007, ApJ, 656, 148 Armus, L., Mazzarella, J. M., Evans, A. S., et al. 2009, PASP, 121, 559 Balestra, I., Boller, T., Gallo, L., Lutz, D., \& Hess, S. 2005, A\&A, 442, 469
Barber, C. R., Roberts, T. P., \& Warwick, R. S. 1996, MNRAS, 282, 157 Bondi, M., Pérez-Torres, M.-A., Dallacasa, D., \& Muxlow, T. W. B. 2005, MNRAS, 361, 748

Carilli, C. L., \& Taylor, G. B. 2000, ApJ, 532, L95

Colina, L., Arribas, S., \& Borne, K. D. 1999, ApJ, 527, L13

Condon, J. J., Helou, G., Sanders, D. B., \& Soifer, B. T. 1990, ApJS, 73, 359

Downes, D., \& Solomon, P. M. 1998, ApJ, 507, 615

Elvis, M., Wilkes, B. J., McDowell, J. C., et al. 1994, ApJS, 95, 1

Genzel, R., Lutz, D., Sturm, E., et al. 1998, ApJ, 498, 579

González-Martín, O., Masegosa, J., Márquez, I., Guerrero, M. A., \& DultzinHacyan, D. 2006, A\&A, 460, 45

Grimes, J. P., Heckman, T., Strickland, D., \& Ptak, A. 2005, ApJ, 628, 187

Heckman, T. M., Armus, L., \& Miley, G. K. 1990, ApJS, 74, 833

Iwasawa, K. 1999, MNRAS, 302, 96

Iwasawa, K., Wilson, A. S., Fabian, A. C., \& Young, A. J. 2003, MNRAS, 345, 369

Iwasawa, K., Sanders, D. B., Teng, S. H., et al. 2011, ApJ, in press

Kaastra, J. S. 1992, Internal SRON-Leiden Report, ver. 2.0

Khachikian, E. Y., \& Weedman, D. W. 1974, ApJ, 192, 581

Kalberla, P. M. W., Burton, W. B., Hartmann, D., et al. 2005, A\&A, 440, 775

Kim, M., Kim, D.-W., Wilkes, B. J., et al. 2007, ApJS, 169, 401

Knapen, J. H., Laine, S., Yates, J. A., et al. 1997, ApJ, 490, L29

Komossa, S., Burwitz, V., Hasinger, G., et al. 2003, ApJ, 582, L15

Lusso, E., Comastri, A., Vignali, C., et al. 2010, A\&A, 512, A34

Majewski, S. R., Hereld, M., Koo, D. C., Illingworth, G. D., \& Heckman, T. M. 1993, ApJ, 402, 125

Marconi, A., Risaliti, G., Gilli, R., et al. 2004, MNRAS, 351, 169

Mazzarella, J. M., \& Boroson, T. A. 1993, ApJS, 85, 27

Mazzarella, J. M., Iwasawa, K., Vavilkin, T., et al. 2011, AJ, submitted

Netzer, H., Lemze, D., Kaspi, S., et al. 2005, ApJ, 629, 739

Nomoto, K., Hashimoto, M., Tsujimoto, T., et al. 1997, Nucl. Phys. A, 616, 79

Petric, A. O., Armus, L., Howell, J., et al. 2010, ApJ, in press [arXiv: 1012.1891$]$

Ptak, A., Heckman, T., Levenson, N. A., Weaver, K., \& Strickland, D. 2003, ApJ, 592, 782

Ranalli, P., Comastri, A., Origlia, L., \& Maiolino, R. 2008, MNRAS, 386, 1464

Risaliti, G., Gilli, R., Maiolino, R., \& Salvati, M. 2000, A\&A, 357, 13

Sanders, D. B., Soifer, B. T., Elias, J. H., et al. 1988, ApJ, 325, 74

Sanders, D. B., Mazzarella, J. M., Kim, D.-C., Surace, J. A., \& Soifer, B. T. 2003, AJ, 126, 1607

Satyapal, S., Sambruna, R. M., \& Dudik, R. P. 2004, A\&A, 414, 825

Scoville, N. Z., Evans, A. S., Thompson, R., et al. 2000, AJ, 119, 991

Soifer, B. T., Neugebauer, G., Matthews, K., et al. 2000, AJ, 119, 509

Teng, S. H., Veilleux, S., Anabuki, N., et al. 2009, ApJ, 691, 261

Veilleux, S., Kim, D.-C., Sanders, D. B., Mazzarella, J. M., \& Soifer, B. T. 1995, ApJS, 98, 171

Veilleux, S., Cecil, G., \& Bland-Hawthorn, J. 2005, ARA\&A, 43, 769

Xia, X.-Y., Boller, T., Wu, H., et al. 1998, ApJ, 496, L9

Xia, X. Y., Xue, S. J., Mao, S., et al. 2002, ApJ, 564, 196 